\title{
Association between Endoglin and nm23-H1 Expression and the Recurrence in Elderly Patients with Laryngeal Squamous Cell Carcinoma
}

\author{
PEI. JU. ZHOU, YUAN YUAN YANG AND YA QIN ZHU* \\ Department of Otolaryngology, The First Affiliated Hospital of Soochow University, No. 188, Shizi Street, Suzhou, Jiangsu \\ 215006, China
}

Zhou et al.: Association between CD105 and nm23-H1 expression and the recurrence in elderly patients

To explore the association between endoglin and $\mathrm{nm} 23-\mathrm{H} 1$ expression and the recurrence in elderly patients with laryngeal squamous cell carcinoma. Patients diagnosed with laryngeal squamous cell carcinoma in The First Affiliated Hospital of Soochow University Suzhou from January 2012 to April 2015 were selected as the subjects, all patients underwent surgical resection and followed up for $5 \mathrm{y}$, and disease recurrence was chosen as the experimental endpoint. The basic clinical data of all patients were collected, immunohistochemical methods was used to evaluate the expression of endoglin and nm23-H1. According to disease recurrence patients were divided into relapse group and non-relapse group, the differences between the two groups were compared. The independent related factors affecting the relapse of patients were investigated by Logistic regression analysis. 32 patients relapsed with a recurrence rate of $31.37 \%$. There were significant differences in $\mathrm{T}$ stage, clinical stage, and pathological grade between the two groups. The positive expression rate of endoglin in the relapse group was significantly higher than that in the non-relapse group $((6.51 \pm 0.64) \%$ vs. $(5.63 \pm 0.57) \%, p<0.001)$; the positive expression rate of nm23-H1 was significantly lower than that in the non-relapse group $((9.71 \pm 0.84) \%$ vs. $(10.52 \pm 0.78) \%)$. Multivariate logistic regression analysis showed that $\mathrm{nm} 23-\mathrm{H1}$ and endoglin were independent related factors that affected the recurrence of the disease. Endoglin expression $\geq 5.91 \%, \mathbf{n m} 23-\mathrm{H} 1$ expression $<10.27 \%$ are independent risk factors that affect patients' relapse.

Key words: Laryngeal squamous cell carcinoma, relapse, CD105, nm23-H1

Laryngeal squamous cell carcinoma (LSCC), as one of the common head and neck malignancies, has an incidence of 2.04/1 00000 in China from 2003 to $2007^{[1]}$. Although the $5 \mathrm{y}$ survival rate of patients with early laryngeal cancer after surgical treatment is relatively high, $60 \%$ of patients with laryngeal cancer are diagnosed as advanced stage at the first time, and the recurrence rate of patients receiving first-line treatment is still as high as $50 \%{ }^{[2,3]}$. In recent $y$, with the continuous progress of clinical treatment technology, the level of diagnosis and treatment of laryngeal cancer has been obviously improved, but recurrence is still an important factor affecting the survival and quality of life of patients. CD105 (endoglin) is not or weakly expressed in vascular endothelial cells of normal mature tissues, while significantly up-regulated in the endothelial cells with proliferation and neovascularization in tumor tissues, which has become a marker of tumor diagnosis, treatment and prognosis ${ }^{[4]}$. Tumor metastasis suppressor gene (NM23) family plays an important role in cell differentiation, proliferation and apoptosis. NM23-H1, a tumor metastasis suppressor gene isolated and identified in 1988, encodes nucleoside diphosphate kinase (NDPK), which plays a mediating role in cell differentiation and signal transduction system $^{[5]}$. In addition, previous study have found that NM23-H1 is the most closely related to tumor invasion and metastasis ${ }^{[6]}$. Present study selected elderly patients with LSCC who received primary tumor resection and radiotherapy in our hospital. Through 5-y follow-up, we recorded the recurrence of patients and explored the independent related factors affecting the recurrence of patients. From January 2012 to April 2015, a total 102 patients with LSCC who were treated in the First Affiliated Hospital of Soochow University and underwent surgical resection were selected as the research objects. Among these patients, 100 were male and 2 were female. The age of patients was (60-79) y, with average of (68.7 \pm 5.4$)$ y. All patients were not given radiotherapy, 
chemotherapy, hormone or immunotherapy before operation. The basic clinical data of all patients were complete. The pathological specimens of tumor tissues were fixed with formaldehyde (10\%) and embedded in paraffin. All patients were followed up for $5 \mathrm{y}$ by outpatient or telephone. The date of diagnosis was taken as the starting point of the study, and the recurrence of the disease was the end point of the study. This study has been approved by the medical ethics committee of our hospital, and all patients have agreed and signed the informed consent. According to the location of laryngeal cancer (UICC TNM staging standard in 2006), the patients were divided into supraglottic type (26 cases) and glottic type $(76 \text { cases })^{[7]}$. T stage: $\mathrm{T} 1=30$ cases, $\mathrm{T} 2=32$ cases, $\mathrm{T} 3=22$ cases, $\mathrm{T} 4=18$ cases; clinical stage: stage $\mathrm{I}=30$ cases, stage $\mathrm{II}=25$ cases, stage $\mathrm{III}=21$ cases, stage IV=26 cases. Pathological grade: high differentiation $=26$ cases, medium differentiation $=50$ cases, low differentiation $=26$ cases. There were 16 cases with cervical lymph node metastasis and 86 cases without cervical lymph node metastasis. Smoking index $=$ number of cigarettes per $\mathrm{d} \times \mathrm{y}$ of smoking. The patients were divided into recurrence group and nonrecurrence group according to whether the patients relapsed at the end of follow-up. Follow up interval: follow up was conducted once a mo in the first $y$, every two mo in the second y, every three mo in the third y, and half a y in the fourth and fifth y. The recurrence of the disease was taken as the end point. Reagents: Mouse anti human cd105 monoclonal antibody and nm23-H1 Rabbit anti human polyclonal antibody were purchased from Wuhan Boshi Company (Boshi, Wuhan, China). Immunohistochemistry and HE staining: The tissue samples were embedded in paraffin, and then sectioned continuously with $4 \mu \mathrm{m}$ thickness. $\mathrm{HE}$ and. Immunohistochemistry staining was performed according to the instructions of the kit. The known positive tablets were used as the control group, and PBS solution was used as the blank control. The slices were read by two pathologists in double-blind way and recorded to determine the results. Positive cell counting method: 5 high power visual fields were observed randomly in each section. The number of positive cells in each field was counted. The average value of positive cells was calculated. SPSS 22.0 was used for statistical analysis in our study. The count data such as age, NM23-H1 positive rate and CD105 positive expression rate were expressed as mean $\pm \mathrm{SD}$. The differences between the two groups were compared by t test. Statistical data such as classification, gender and clinical stage of laryngeal carcinoma were expressed by rate. Chi square test was used for the difference between the three or more groups. Logistic regression analysis was used to explore the related factors affecting the recurrence of patients, $\mathrm{P}<0.05$ was considered to be statistically significant. At the end of the experiment, 32 patients had tumor recurrence; the recurrence rate was $31.37 \%$. There was no significant difference in age, sex ratio, classification of laryngeal cancer, cervical lymph node metastasis and smoking index between the two groups. In the recurrence group, the proportion of patients with stage $(\mathrm{I}+\mathrm{II})$ in $\mathrm{T}$ stage was markedly lower than that in the non-recurrence group, and in the clinical stage, $(\mathrm{I}+\mathrm{II})$ was also obviously lower than that in the non-recurrence group. In the pathological grading, the proportion of highly differentiated patients was remarkably lower than that of the non-recurrence group. The average positive expression rate of cd105 in recurrent group was $(6.51 \pm 0.64) \%$, which was significantly higher than that in non-recurrence group $(5.63 \pm 0.57) \%$; the average positive expression rate of nm23-H1 was $(9.71 \pm 0.84) \%$, which was significantly lower than that of non-recurrence group $(10.52 \pm 0.78)$ $\%$. Among all the subjects, the average positive expression rate of cd105 was $(5.91 \pm 0.63) \%$, and the average positive expression rate of $\mathrm{nm} 23-\mathrm{H} 1$ was $(10.27 \pm 0.86) \%$. According to the mean value, the proportion of cd105 positive expression value above the mean value in relapse group was significantly higher than that in non-recurrence group, and the proportion of $\mathrm{nm} 23-\mathrm{H} 1$ positive expression value above the average value in recurrent group was significantly less than that in non-recurrence group, as shown in Table 1. The univariate logistic regression analysis results indicated that $\mathrm{T}$ stage, clinical stage, pathological grade, CD105 positive expression value and NM23-H1 positive expression value were significantly related factors influencing the recurrence of patients, as shown in Table 2. Multivariate logistic regression analysis clarified that $\mathrm{CD} 105$ positive expression level and nm23-H1 positive expression level were independent related factors influencing recurrence, $\mathrm{CD} 105$ positive expression value $<5.91 \%$ was independent protective factor of recurrence, $\mathrm{nm} 23-\mathrm{H} 1$ positive expression value $<10.27 \%$ was independent risk factor of recurrence, as shown in Table 3. In this study, the 5-y recurrence rate of LSCC was $31.37 \%$, which was consistent with the conclusion reported in study ${ }^{[8]}$. By logistic regression analysis, we explored the independent factors influencing the recurrence of LSCC. The results showed that CD105 positive expression rate $<5.91 \%$ was an independent protective factor for recurrence, 


\begin{tabular}{|c|c|c|c|c|}
\hline & $\begin{array}{c}\text { Recurrence group } \\
(\mathrm{n}=32)\end{array}$ & $\begin{array}{c}\text { No recurrence group } \\
(n=70)\end{array}$ & $\begin{array}{c}\text { Statistical } \\
\text { value }\end{array}$ & $\mathrm{P}$ value \\
\hline Age (years) & $68.9 \pm 5.7$ & $68.7 \pm 5.5$ & 0.167 & 0.867 \\
\hline Gender (male / female) & $32 / 1$ & $68 / 1$ & 0.080 & 0.781 \\
\hline $\begin{array}{l}\text { Classification of } \\
\text { Laryngeal carcinoma (n, \%) }\end{array}$ & & & 0.17 & 0.68 \\
\hline Supraglottic type & $9(28.13 \%)$ & $17(24.29 \%)$ & & \\
\hline Glottic & $23(71.88 \%)$ & $53(75.71 \%)$ & & \\
\hline T stage & & & 5.676 & 0.017 \\
\hline $1+I I$ & $14(43.75 \%)$ & $48(68.57 \%)$ & & \\
\hline III+IV & $18(56.25 \%)$ & $22(31.43 \%)$ & & \\
\hline Clinical stages & & & 5.061 & 0.024 \\
\hline I+II & $12(37.50 \%)$ & $43(61.43 \%)$ & & \\
\hline III+IV & $20(62.50 \%)$ & $27(38.57 \%)$ & & \\
\hline Pathological grading & & & 4.143 & 0.042 \\
\hline High differentiation & $4(12.50 \%)$ & $22(31.43 \%)$ & & \\
\hline $\begin{array}{l}\text { Medium and } \\
\text { low differentiation }\end{array}$ & $28(87.50 \%)$ & $48(68.57 \%)$ & & \\
\hline Lymph node metastasis & & & 0.331 & 0.565 \\
\hline Yes & $6(18.75 \%)$ & $10(14.29 \%)$ & & \\
\hline No & $26(81.25 \%)$ & $60(85.71 \%)$ & & \\
\hline Smoking index & & & 0.003 & 0.96 \\
\hline$\geq 400$ & $14(43.75 \%)$ & $31(44.29 \%)$ & & \\
\hline$<400$ & $18(56.25 \%)$ & 39 (55.71\%) & & \\
\hline CDI05 positive rate (\%) & $6.51 \pm 0.64$ & $5.63 \pm 0.57$ & 6.959 & $<0.001$ \\
\hline $\mathrm{nm} 23-\mathrm{H} 1$ positive rate $(\%)$ & $9.71 \pm 0.84$ & $10.52 \pm 0.78$ & 4.75 & $<0.001$ \\
\hline $\begin{array}{l}\text { mean CDI05 positive } \\
\text { expression }(n, \%)\end{array}$ & & & 7.607 & 0.006 \\
\hline$\geq$ mean value & $24(75.00 \%)$ & $32(45.71 \%)$ & & \\
\hline$<$ mean value & $8(25.00 \%)$ & $38(54.29 \%)$ & & \\
\hline $\begin{array}{l}\text { mean } n m 23-\mathrm{H} 1 \text { positive } \\
\text { expression }(n, \%)\end{array}$ & & & 6.391 & 0.011 \\
\hline$\geq$ mean value & $12(37.50 \%)$ & $45(64.29 \%)$ & & \\
\hline$<$ mean value & $25(35.71 \%)$ & & & \\
\hline
\end{tabular}

TABLE 2: UNIVARIATE LOGISTIC REGRESSION ANALYSIS OF RECURRENCE IN PATIENTS WITH LSCC

\begin{tabular}{lccccc}
\hline Independent variable & B value & S.E. value & wald $\mathrm{x}^{2}$ value & OR value $(95 \% \mathrm{Cl})$ & $\mathrm{P}$ value \\
\hline T stage & 1.762 & 0.816 & 4.315 & $1.106(1.042-1.267)$ & 0.043 \\
Clinical stages & 0.946 & 0.517 & 4.682 & $1.136(1.072-1.483)$ & 0.032 \\
Pathological grading & 2.016 & 0.618 & 4.508 & $1.226(1.106-1.626)$ & 0.038 \\
CDI05 positive value & 1.215 & 0.826 & 7.615 & $0.764(0.605-0.894)$ & $<0.001$ \\
nm23-H1 positive value & 1.326 & 0.659 & 6.594 & $2.318(1.654-4.167)$ & $<0.001$ \\
\hline
\end{tabular}

Note: T stage: with (I+II) as reference; clinical stage with (I+II) as reference; pathological grading with "high differentiation" as reference; cdl05 positive expression value with " $\geq$ mean $(\geq 5.91 \%) "$ as reference; nm23-H1 positive expression value with " $\geq$ mean value $(\geq 10.27 \%) "$ as reference.

TABLE 3: MULTIVARIATE LOGISTIC REGRESSION ANALYSIS OF RECURRENCE IN PATIENTS WITH LSCC

\begin{tabular}{lccccc}
\hline Independent variable & B value & S.E. value & wald $\mathrm{x}^{2}$ value & OR value $(95 \% \mathrm{Cl})$ & $\mathrm{p}$ value \\
\hline CDI05 positive value & 2.054 & 0.619 & 6.859 & $0.792(0.676-0.925)$ & $<0.001$ \\
nm23-H1 positive value & 1.846 & 0.846 & 5.904 & $1.958(1.322-4.506)$ & $<0.001$ \\
\hline
\end{tabular}

Note: the positive expression value of cdl05 was referred to as " $\geq$ mean value ( $\geq 5.91 \%) " ; n m 23-\mathrm{H} 1$ positive expression value was referred to as " $\geq$ mean value $(\geq 10.27 \%)$ ". 
and $\mathrm{nm} 23-\mathrm{H} 1$ positive expression value $<10.27 \%$ was an independent risk factor for recurrence. CD105, with a molecular weight of $180 \mathrm{KD}$, is a marker of endothelial cell proliferation. It is a component of the transforming growth factor beta superfamily receptor complex ${ }^{[9]}$, which is formed by the polymerization of two monomer disulfide bonds, and is significantly up-regulated in most solid tumors. Previous study ${ }^{[10]}$ had showed that the micro vessel density of CD105 in the stroma of ovarian cancer cells was highly specific, and CD105 was significantly correlated with tumor proliferation, metastasis and prognosis of patients. In addition, CD105 was significantly increased in esophageal squamous cell carcinoma and was an independent prognostic factor ${ }^{[11]}$. Another study ${ }^{[12]}$ showed that the expression level of endothelial CD105 was markedly elevated in patients with cervical lymph node metastasis in primary LSCC, suggesting that CD105 can be used as a predictor of high recurrence risk or poor overall prognosis. The results of a cohort study ${ }^{[13]}$ demonstrated that patients with LSCC with higher CD105 expression had higher local recurrence rate and lower survival rate. In this study, the expression of CD105 in patients with recurrence was significantly higher than that in patients without recurrence. Taking the mean value of CD105 as the dividing line, we found that the percentage of patients with recurrent CD105 exceeding the mean value was significantly higher than that of nonrecurrence group. In addition, univariate and multivariate logistic regression analysis showed that the low expression of CD105 was an independent factor affecting the recurrence of patients, which was consistent with the conclusion reported in the literature. $\mathrm{NM} 23$ gene, located at $17 \mathrm{q} 2 \mathrm{~h}$ on the long arm of chromosome 17, mainly encodes nuclear and cytoplasmic proteins with a molecular weight of $17 \mathrm{KD}^{[14]}$. Nm23 family mediates the regulation of cell proliferation and differentiation, and has the effect of inhibiting tumor metastasis, which may be related to the involvement of nm23 protein in vesicle transport, microtubule polymerization and depolymerization, and signal transduction ${ }^{[15]}$. A report ${ }^{[16]}$ found that tumor suppressor $\mathrm{p} 53$, which is related to the proportion of G1 phase of cell cycle, may be a regulatory factor of $\mathrm{nm} 23-\mathrm{H} 1$. Human hepatoma cell line J7B and breast cancer cell line MCF7 wild-type p53 unregulated the expression of $\mathrm{nm} 23-\mathrm{H} 1$ through mRNA and protein levels, and inhibited the invasion ability of J7B and MCF7. Our results show that the positive rate of $\mathrm{nm} 23-\mathrm{H} 1$ in recurrent patients is significantly lower than that in non-recurrent patients, and the low expression level of $\mathrm{nm} 23-\mathrm{H} 1$ is an independent risk factor for recurrence. Laryngeal squamous cell carcinoma (LSCC) accounts for approximately $18 \%$ of all primary head and neck cancers (HNSCC) in the developed nations across the globe. The positive rate of nm23-H1 in patients with recurrent LSCC was significantly lower, and the results of multivariate regression analysis showed that $\mathrm{nm} 23-\mathrm{H} 1$ was an independent related factor affecting the prognosis of patients, which was consistent with the previous research conclusions ${ }^{[17]}$. Nm23-H1 $<10 \%$ and CD105 $\geq 6 \%$ were independent risk factors for LSCC recurrence, which were consistent with a previous study ${ }^{[18]}$. As a prospective study, we followed LSCC patients for $5 \mathrm{y}$. The preliminary results showed that nm23-H1 and CD105 were independent related factors of recurrence. However, the subjects included in this study are limited, and the follow-up time is short. The recurrence of the disease is taken as the end point of the experiment, and the related factors affecting the survival time of the patients have not been further studied. Large sample size is needed to predict the recurrence of cancer by nm23-H1 and CD105 positive levels. To further study the independent related factors of recurrence and prognosis of image LSCC patients, we should expand the sample size to further study and analyze comprehensively. In conclusion, $\mathrm{nm} 23-\mathrm{H} 1<10.26 \%$ and $\mathrm{CD} 105 \geq 5.91$ are independent risk factors for LSCC recurrence.

\section{Acknowledgement:}

This work was supported by The First Affiliated Hospital of Soochow University.

\section{Conflict of interests:}

The authors declared no conflict of interest.

\section{REFERENCES}

1. Li Li, Ma Shiyuan. Quality of life and influencing factors of postoperative laryngeal cancer patients. Chin J Gerontol 2016;36:2028-30.

2. Anschuetz L, Shelan M, Dematté M, Schubert AD, Giger R, Elicin O. Long-term functional outcome after laryngeal cancer treatment. Radiat Oncol 2019;14:101.

3. Hu Xiaoqin, Wu Keming, Zheng Guofeng. Long term efficacy and prognostic factors of elderly patients with laryngeal cancer. Chin J GerontoL 2017;37:2972-4.

4. Metcalfe E, Arik D, Oge T, Etiz D, Yalcin OT, Kabukcuoglu $\mathrm{S}$, et al. CD105 (endoglin) expression as a prognostic marker of angiogenesis in squamous cell cervical cancer treated with radical radiotherapy. J Cancer Res Ther 2018;14:1373-8.

5. Marioni G, Ottaviano G, de Filippis C, Fasanaro E, Randon B, Meneghesso S, et al. Nuclear expression of onco-suppressors $\mathrm{nm} 23-\mathrm{H} 1$ and maspin are associated with lower recurrence rate in laryngeal carcinoma. Am J Otolaryngol 2019;40:224-9. 
6. Keshavarz-Pakseresht B, Shandiz SA, Baghbani-Arani F. Imatinib induces up-regulation of NM23, a metastasis suppressor gene, in human Hepatocarcinoma (HepG2) Cell Line. Gastroenterol Hepatol Bed Bench 2017;10:29-33.

7. Li Na. Analysis of the clinical efficacy of surgical treatment in 112 patients with laryngeal cancer. Dalian Medical University, 2018:1-40

8. Fong PY, Tan SH, Lim DW, Tan EH, Ng QS, Sommat K, et al. Association of clinical factors with survival outcomes in laryngeal squamous cell carcinoma (LSCC). PLoS One 2019;14:e0224665.

9. Jamshidi S, Zargaran M, Roshanaei G, Hadadi F, Dehghani A. Immunohistochemical Comparison of the Expression of CD34 and CD105 in Odontogenic Keratocyst and Dentigerous Cyst. J Dent 2017;18:43-6.

10. Zhang J, Yuan B, Zhang H, Li H. Human epithelial ovarian cancer cells expressing CD105, CD44 and CD106 surface markers exhibit increased invasive capacity and drug resistance. Oncol Lett, 2019;17:5351-60.

11. Kumagai Y, Sobajima J, Higashi M, Ishiguro T, Fukuchi M, Ishibashi $\mathrm{K}$, et al. Angiogenesis in superficial esophageal squamous cell carcinoma: assessment of microvessel density based on immunostaining for CD34 and CD105. Jpn J Clin Oncol 2014;44:526-33.

12. Su J, Ji XB, Xie JH, Li W. Expressions of angiogenesis-related factors: CD105, EphA2 and EphrinA1 in laryngeal squamous cell carcinoma and clinical implication. Zhonghua Er Bi Yan Hou Tou Jing Wai Ke Za Zhi 2016;51:929-36.

13. Lovato A, Marioni G, Manzato E, Staffieri C, Giacomelli L, Ralli G, et al. Elderly patients at higher risk of laryngeal carcinoma recurrence could be identified by a panel of two biomarkers (nm23-H1 and CD105) and $\mathrm{pN}^{+}$status. Eur Arch Otorhinolaryngol 2015;272:3417-24.

14. Lee JJ, Kim HS, Lee JS, Park J, Shin SC, Song S, et al. Small molecule activator of Nm23/NDPK as an inhibitor of metastasis. Sci Rep 2018;8:1-3.

15. Wu M, Yu G, Yan T, Ke D, Wang Q, Liu R, et al. Phosphorylation of SET mediates apoptosis via P53 hyperactivation and NM23-H1 nuclear import. Neurobiol Aging 2018;69:38-47.

16. Liu L, Li M, Zhang C, Zhang J, Li G, Zhang Z, et al. Prognostic value and clinicopathologic significance of $n m 23$ in various cancers: A systematic review and meta-analysis. Int J Surg 2018;60:257-65.

17. Zheng Z, Tian R, Wang P. Roles of KAI1 and nm23 in lymphangiogenesis and lymph metastasis of laryngeal squamous cell carcinoma. World J Surg Oncol 2017;15:211.

18. Franz L, Tealdo G, Contro G, Bandolin L, Carraro V, Giacomelli $\mathrm{L}$, et al. Biological tumor markers (maspin, CD105, nm23-H1) and disease relapse in laryngeal cancer: cluster analysis. Head Neck 2020.

This is an open access article distributed under the terms of the Creative Commons Attribution-NonCommercial-ShareAlike 3.0 License, which allows others to remix, tweak, and build upon the work non-commercially, as long as the author is credited and the new creations are licensed under the identical terms

$\begin{aligned} & \text { This article was originally published in a special issue, } \\ & \text { "Biomedical Research in Clinical and Preclinical } \\ & \text { Pharmaceutics" Indian J Pharm Sci 2020:82(5)Spl } \\ & \text { issue7;152-156 }\end{aligned}$

